\title{
SIMPLE ENVIRONMENT FOR DEVELOPING METHODS OF CONTROLLING CHAOS IN SPATIALLY DISTRIBUTED SYSTEMS
}

\author{
ŁUKASZ KORUS \\ Institute of Informatics, Automatics and Robotics \\ Wrocław University of Technology, ul. Janiszewskiego 11/17, 50-370 Wrocław, Poland \\ e-mail: lukasz.korus@pwr.wroc.pl
}

\begin{abstract}
The paper presents a simple mathematical model called a coupled map lattice (CML). For some range of its parameters, this model generates complex, spatiotemporal behavior which seems to be chaotic. The main purpose of the paper is to provide results of stability analysis and compare them with those obtained from numerical simulation. The indirect Lyapunov method and Lyapunov exponents are used to examine the dependence on initial conditions. The net direction phase is introduced to measure the symmetry of the system state trajectory. In addition, a real system, which can be modeled by the CML, is presented. In general, this article describes basic elements of environment, which can be used for creating and examining methods of chaos controlling in systems with spatiotemporal dynamics.
\end{abstract}

Keywords: coupled map lattice, spatiotemporal chaos, system stability, Lyapunov exponents, net direction phase.

\section{Introduction}

Deterministic chaos is complex behavior which can be generated by a nonlinear dynamic system as a result of state trajectory evolution when its previous history is known (Ott, 2002). The origin of this phenomenon in nonlinear dynamic systems is connected with their sensitivity to initial conditions. In fact, chaotic behavior is a common phenomenon discovered in physics (liquids (Procaccia and Meron, 1986; Langenberg et al., 2004), lasers (Yamada and Graham, 1980; Used and Martin, 2010), plasma (Held et al., 1984; Banerjee et al., 2010)), mechanics (Chui and Ma, 1982; Ott, 2002), electronics (Gunaratne et al., 1989; Yim et al., 2004), chemistry (Argoul et al., 1987; Córdoba et al., 2006), biology (action of human heart (Govindan et al., 1998) and brain (Andrzejak et al., 2001; Gautama et al., 2003)), and in many other branches of science and industry.

Chaotic systems, due to their sensitivity to initial conditions, are unpredictable but, as has been proved, they can be effectively controlled (Ott et al., 1990; Singer et al., 1991; Dressler and Nitsche, 1992; Chen and Dong, 1993; Alsing et al., 1994; Pyragas, 2001; Andrievskii and Fradkov, 2003). The control of chaos in such a type of system is considered a process which changes an irregular and unpredictable behavior to a well-ordered and periodical one. Sensitivity to initial conditions is par- ticularly important in real, physical systems. For example, a chaotic behavior in chemical reactors can cause unpredictable and negative results. Moreover, many real systems and their mathematical models represent similar spatiotemporal behaviors, where chaos can be observed both in time and space. As has been shown, chaos in spatially distributed dynamical systems can also be controlled (Auerbach, 1994; Astakhov et al., 1995; Parmananda, 1997; Parekh et al., 1998; Zhu and Chen, 2001; Mihaliuk et al., 2002; Beck et al., 2002; Boukabou and Mansouri, 2005). This makes this issue even more essential and it seems that it could have numerous practical applications (i.e., chemical reactors, lasers, plasma).

The main purpose of the paper is to provide results of both analytical and numerical research regarding CML system stability. In addition, we found it very important to chose and present proper tools which can be useful during the examination of the system sensitivity to initial conditions. Based on measurements of this property and the level of state trajectory symmetry, some conclusions about the existence of chaos in the system can be made.

The presented model and tools can be helpful while creating and checking new algorithms of controlling chaos for spatiotemporal systems. It is even more essential as its potential, physical application is presented. In general, this article describes basic elements of environment which can be used for creating and examining methods of con- 
Table 1. Classification of systems with spatiotemporal dynamics.

\begin{tabular}{|l|c|c|c|}
\hline Model & Space & Time & Local structure \\
\hline \hline Partial differential equations (PDEs) & $\mathrm{C}$ & $\mathrm{C}$ & $\mathrm{C}$ \\
\hline Iterated functional equations (IFEs) & $\mathrm{C}$ & $\mathrm{D}$ & $\mathrm{C}$ \\
\hline Oscillator chains (OCs) & $\mathrm{D}$ & $\mathrm{C}$ & $\mathrm{C}$ \\
\hline Lattice dynamical systems (LDSs) & $\mathrm{D}$ & $\mathrm{D}$ & $\mathrm{C}$ \\
\hline Cellular automata (CA) & $\mathrm{D}$ & $\mathrm{D}$ & $\mathrm{D}$ \\
\hline
\end{tabular}

trolling chaos in systems with spatiotemporal dynamics.

\section{Models of systems with spatiotemporal dynamics}

2.1. Classification. The classification of mathematical methods used for modeling systems with spatiotemporal dynamics was presented by Crutchfield and Kaneko (1987). This classification is based on choices of the discretization of space, time and local state variables (see Table 1).

The letter ' $\mathrm{C}$ ' in particular columns of Table 1 stands for continuous values while the letter ' $\mathrm{D}$ ' for discrete values. The table contains five most frequently used models for systems with spatiotemporal dynamics. For instance, partial differential equations (PDEs) require a continuous function of time and space to specify a state. Lattice dynamic systems (LDSs) and cellular automata (CA) (Weimar, 1997; Chopard et al., 2002; Jacewicz, 2002) are constructed using discrete lattices $(N$-dimensional in general) and their states change according to a discrete clock. The difference between LDSs and CA results from different types of values in each node of lattice. In LDSs, the set of states is infinite and contains real values, while in CA only integers are allowed. The LDS class includes also the coupled map lattice, i.e., the model which is presented in this article.

When the distribution of nodes in the lattice is considered, CA and the CML can be put into a single common class known as spatially distributed dynamic systems with a regular structure. This class is special, because there is a symmetry of local connections for all lattice nodes. This symmetry remains unchanged for the whole system.

2.2. Brief characteristic of classes. Real-valued state variables in the CML make this model more efficient than CA when it comes to the modeling of real systems. Even for a small lattice size, behaviors similar to real ones can be observed (Kaneko, 1990). In CA, each cell is treated as a macro-particle, therefore a bigger lattice size is necessary to perform a real simulation (Weimar, 1997; Chopard et al., 2002; Jacewicz, 2002; Korus, 2007). For both types of systems, i.e., CA and the CML, principles of dynamic systems theory and information theory can be applied. Because of complex behaviors obtained in CMLs even for small lattice sizes, this model is frequently used for modeling chaos. In addition, it is worth stating that both CA and CMLs are easy to implement as computer algorithms, mainly due to their simple structure. In practice, it is much easier to implement CA or CMLs than to use PDEs on computers.

To sum up, it has to be highlighted that CMLs can be used as simple dynamic systems where complex collective behaviors, including spatiotemporal chaos, may be observed.

\section{CML: Model definition}

A single-dimensional coupled map lattice can be considered as a set of simple components numbered as $n=$ $1, \ldots, N$. The state of each component given by $x_{t}(n)$ changes in discrete time steps: $t=1, \ldots, T$. It is calculated based on state values from the previous time step in surrounding components according to the evolution function (Crutchfield and Kaneko, 1987; Kaneko, 1990):

$$
\begin{aligned}
x_{t+1}(n)= & f\left(x_{t}(n)\right)+\varepsilon_{L} g\left(x_{t}(n-1)\right) \\
& +\varepsilon_{0} g\left(x_{t}(n)\right)+\varepsilon_{R} g\left(x_{t}(n+1)\right),
\end{aligned}
$$

where the vector

$$
\varepsilon=\left(\varepsilon_{L}, \varepsilon_{0}, \varepsilon_{R}\right)
$$

is the coupling kernel. The function $f$ defines local dynamics and it is the most often taken as a logistic map

$$
f(x)=p x(1-x),
$$

or a circular map

$$
f(x)=\omega+x+k \sin (2 \pi x) .
$$

The function $g$ defines coupling dynamics and may be selected as the linear coupling

$$
g(x)=x
$$

or the same as a local function

$$
g(x)=f(x) .
$$

The system states in a particular time step can be collected using the vector

$$
\mathbf{x}_{t}=\left(x_{t}(1), x_{t}(2), \ldots, x_{t}(N)\right) .
$$


The paper presents examination results for the CML with diffusive coupling. The kernel for this system is given by the vector

$$
\varepsilon=\left(\varepsilon_{L}, \varepsilon_{0}, \varepsilon_{R}\right)=\left(\frac{\varepsilon}{2}, 1-\varepsilon, \frac{\varepsilon}{2}\right)
$$

Replacing the coupling kernel from the general evolution function (11) with (8) and removing the part representing local dynamics, the evolution function of the system can be written as

$$
\begin{aligned}
x_{t+1}(n)= & (1-\varepsilon) g\left(x_{t}(n)\right) \\
& +\frac{\varepsilon}{2}\left[g\left(x_{t}(n-1)\right)+g\left(x_{t}(n+1)\right)\right],
\end{aligned}
$$

where the coupling function is given by the logistic equation

$$
g(x)=p x(1-x) .
$$

A detailed analysis of transition to chaos in the logistic equation can be found in the work of Ott (2002).

At the beginning of simulation, i.e., for the time step $t=1$, states values for all components are generated from a uniform distribution. The boundary conditions depend on the modeled problem and can be chosen as fixed, periodic, free, etc. In this paper, a CML model with fixed boundary conditions

$$
\begin{aligned}
x(N+1) & =0, \\
x(0) & =0
\end{aligned}
$$

is examined.

Figure 1 presents the structure of a singledimensional coupled map lattice. Figure 2 contains three diagrams which show the behavior of the CML in time and space domains for $\varepsilon=0.8$ and $p=4$. Chaotic properties of the coupling function, which is the logistic equation in this case, are additionally strengthened in the CML by couplings with surrounding components.

\section{Detection of chaotic behavior}

In this section, two coefficients which provide quantitative evaluation of chaotic properties are described.

4.1. Lyapunov exponents. Lyapunov exponents provide quantitative information about the separation rate of preliminarily close trajectories (Ott, 2002). For single-dimensional discrete equations given by the formula $x_{t+1}=g\left(x_{t}\right)$, we have

$$
h=\lim _{T \rightarrow \infty} \frac{1}{T} \sum_{t=1}^{T} \ln \left|g^{\prime}\left(x_{t}\right)\right| .
$$

The positive Lyapunov exponent means that chaos is present.

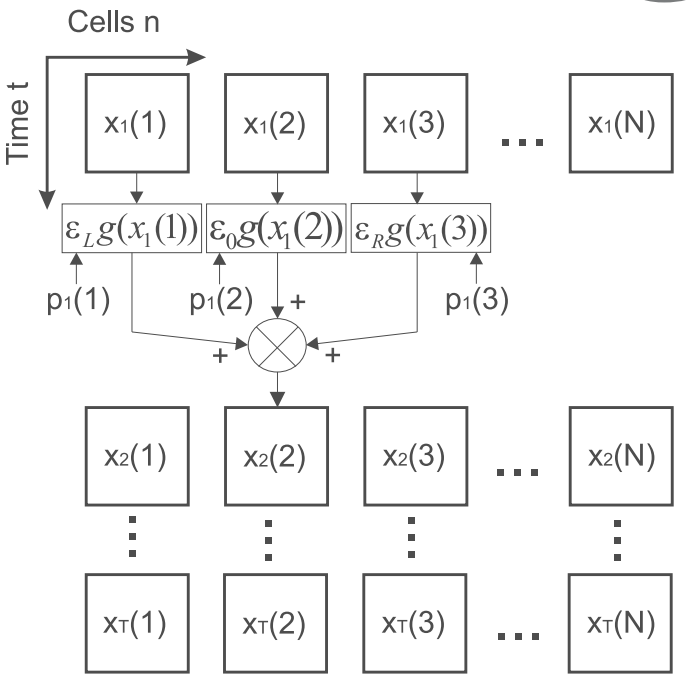

Fig. 1. Structure of the coupled map lattice under examination.

4.2. Net direction phase. The net direction phase introduced by Wei et al. (2000) is an indicator similar to magnetization, which can be defined as

$$
M=\frac{1}{T} \sum_{t=1}^{T} S(t),
$$

where $S(t)=1$ for $x_{t+1}-x_{t} \geq 1$ and $S(t)=-1$ for $x_{t+1}-x_{t}<1$, and $T$ is the iteration number. The direction $S(t)=1$ can be denoted by $S_{\uparrow}$ and $S(t)=-1$ as $S_{\downarrow}$. Whenever $M=0$, the situation can be described as the zero net direction phase. This means that the system trajectory $x_{t}$ switches symmetrically from the upward direction phase $\left(S_{\uparrow}\right)$ to downward direction phase $\left(S_{\downarrow}\right)$ and the system behaves regularly. If $M \neq 0$, this means that the arrangement of the upward and downward direction phases is disordered and the system generates non-periodical trajectories.

The bifurcation diagram and diagrams of the Lyapunov exponent and the net direction phase versus parameter $p$ for the logistic equation 10 are shown in Fig. 3

\section{CML: Stability analysis}

This section contains results of stability analysis for the CML model given by (9). The analysis was performed using the indirect Lyapunov method for non-linear dynamic systems. The method is called indirect because conclusions on the stability of a nonlinear dynamic system are formulated based on results of stability analysis obtained for its linear approximation. Consider a discrete dynamic system

$$
\mathbf{y}_{t+1}=F\left(\mathbf{y}_{t}\right),
$$

where $\mathbf{y}_{t}$ and $\mathbf{y}_{t+1}$ are consecutive state vectors. The first step of the method is to form a linear approximation of the 


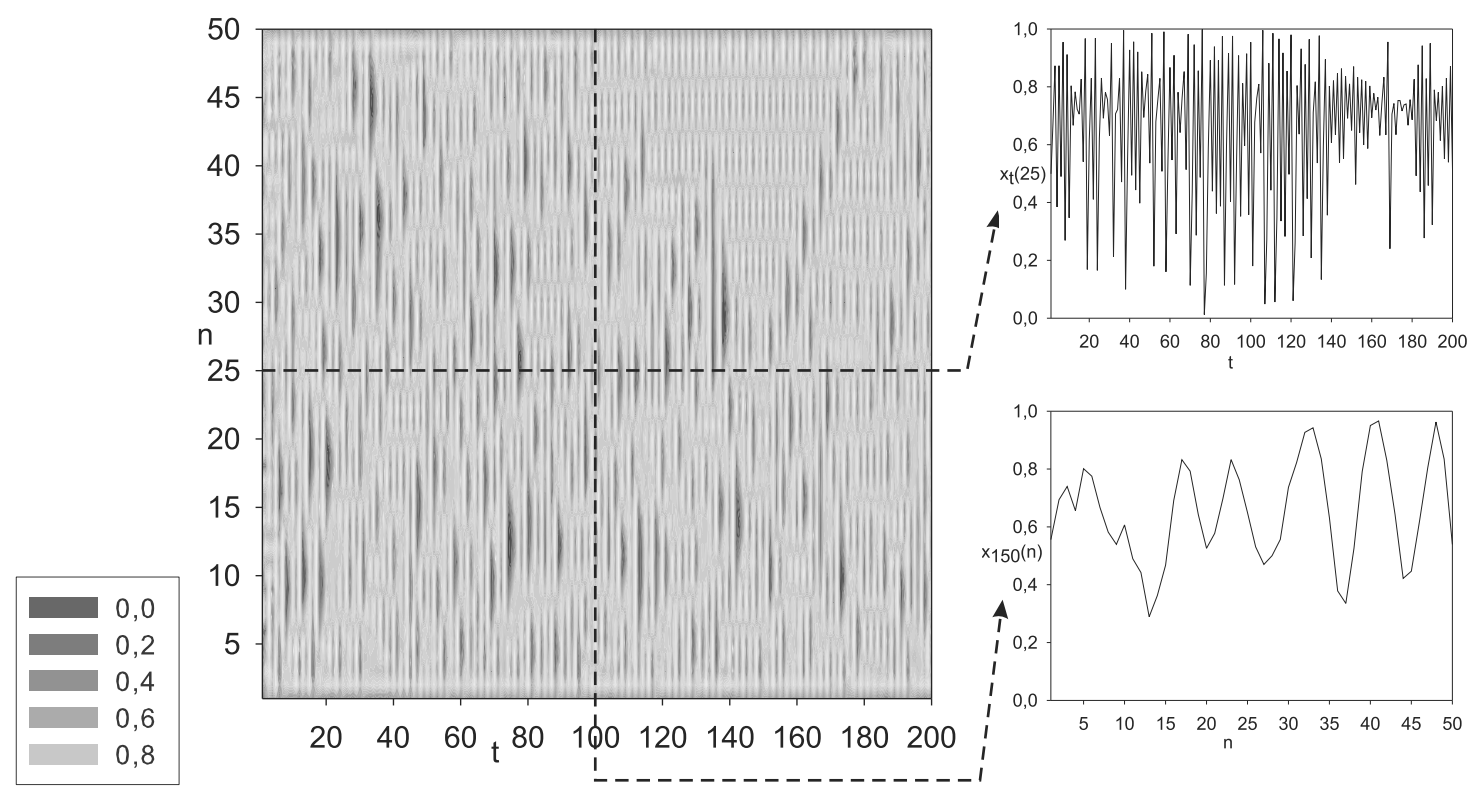

Fig. 2. Diagram illustrating the lattice behavior in time and space domains for $\varepsilon=0.8$ and $p=4$.
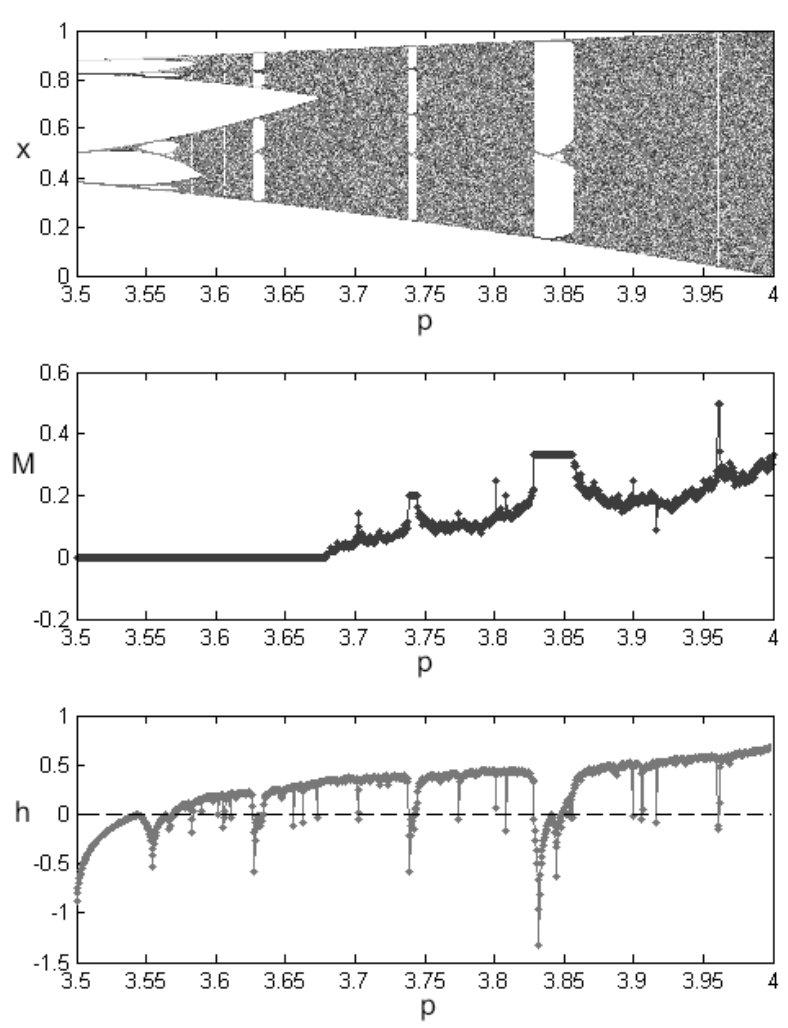

Fig. 3. Bifurcation diagram $x(p)$, the net direction phase $M$ and the Lyapunov exponent $h$ versus the parameter of the logistic equation $p$. original nonlinear system:

$$
\mathbf{y}_{t+1}=J \mathbf{y}_{t}+r
$$

where $J=\partial F_{i} / \partial y_{j}$ is a Jacobi matrix and $r$ is the infinitesimal remainder, satisfying

$$
\lim _{\mathbf{y}_{\mathbf{t}} \rightarrow 0} \frac{r\left(\mathbf{y}_{t}\right)}{\left\|\mathbf{y}_{\mathbf{t}}\right\|}=0 .
$$

Thus, the nonlinear system (14) can be written as linearization

$$
\mathbf{y}_{t+1} \approx J \mathbf{y}_{t}
$$

where $J$ is the Jacobi matrix determined at a fixed point $y_{F}$. The next step is to examine the stability of the linear approximation and to draw conclusions concerning the nonlinear system. According to the Lyapunov method, a nonlinear system is locally stable asymptotically when its linear approximation is asymptotically stable at this point. A nonlinear system is unstable if so is its linear approximation. However, if the linear approximation is stable, but not necessarily asymptotically, nothing can be concluded about the stability of a the original nonlinear system.

The general evolution equation (9) can be also written in matrix form:

$$
\mathbf{x}_{t+1}=W G\left(\mathbf{x}_{t}\right),
$$

where

$$
\begin{aligned}
\mathbf{x}_{t+1} & =\left[x_{t+1}(1) \ldots, x_{t+1}(N)\right]^{T}, \\
\mathbf{x}_{t} & =\left[x_{t}(1), \ldots, x_{t}(N)\right]^{T},
\end{aligned}
$$




$$
W=\left[\begin{array}{ccccc}
(1-\varepsilon) & \frac{\varepsilon}{2} & 0 & \ldots & 0 \\
\frac{\varepsilon}{2} & (1-\varepsilon) & \frac{\varepsilon}{2} & \ldots & 0 \\
0 & \frac{\varepsilon}{2} & (1-\varepsilon) & \ldots & 0 \\
\vdots & \vdots & \vdots & \ddots & \vdots \\
0 & 0 & 0 & \ldots & (1-\varepsilon)
\end{array}\right]_{(20)}
$$

The linearization of such a system around a fixed point $x_{F}$, can be written as follows:

$$
\mathbf{e}_{t+1} \approx J\left(x_{F}\right) \mathbf{e}_{t},
$$

where

$$
\begin{gathered}
\mathbf{e}_{t+1}=\left[x_{t+1}(1)-x_{F}, \ldots, x_{t+1}(N)-x_{F}\right]^{T}, \\
\mathbf{e}_{t}=\left[x_{t}(1)-x_{F}, \ldots, x_{t}(N)-x_{F}\right]^{T}, \\
J=\left[\begin{array}{cccc}
(1-\varepsilon) \Lambda & \frac{\varepsilon}{2} \Lambda & \ldots & 0 \\
\frac{\varepsilon}{2} \Lambda & (1-\varepsilon) \Lambda & \ldots & 0 \\
0 & \frac{\varepsilon}{2} \Lambda & \ldots & 0 \\
\vdots & \vdots & \ddots & \vdots \\
0 & 0 & \ldots & (1-\varepsilon) \Lambda
\end{array}\right],
\end{gathered}
$$

with $\Lambda=g^{\prime}\left(x_{F}\right)$. Assuming that $A=(1-\varepsilon) \Lambda$ and $B=(\varepsilon / 2) \Lambda$, the Jacobi matrix can be written as

$$
J=\left[\begin{array}{cccccc}
A & B & 0 & \ldots & 0 & 0 \\
B & A & B & \ldots & 0 & 0 \\
0 & B & A & \ldots & 0 & 0 \\
\vdots & \vdots & \vdots & \ddots & \vdots & \vdots \\
0 & 0 & 0 & \ldots & A & B \\
0 & 0 & 0 & \ldots & B & A
\end{array}\right]
$$

While determining the eigenvalues of $J\left(x_{F}\right)$, it is worth using the transformation (Kwon et al., 1997)

$$
J=\Lambda(I-\varepsilon(I+\widehat{J})),
$$

where $I$ is the $N$-dimensional identity matrix and

$$
\widehat{J}=\left[\begin{array}{cccccc}
0 & -\frac{1}{2} & 0 & \ldots & 0 & 0 \\
-\frac{1}{2} & 0 & -\frac{1}{2} & \ldots & 0 & 0 \\
0 & -\frac{1}{2} & 0 & \ldots & 0 & 0 \\
\vdots & \vdots & \vdots & \ddots & \vdots & \vdots \\
0 & 0 & 0 & \ldots & 0 & -\frac{1}{2} \\
0 & 0 & 0 & \ldots & -\frac{1}{2} & 0
\end{array}\right] .
$$

The eigenvalues of the matrix $J$ are

$$
\lambda_{i}=\Lambda\left(1-\varepsilon\left(1+\widehat{\lambda_{i}}\right)\right),
$$

where $\widehat{\lambda_{i}}$ are the eigenvalues of the matrix $\widehat{J}$. They can be determined from the formula (Kwon et al., 1997)

$$
\widehat{\lambda_{i}}=\cos \left(\frac{i \pi}{N}\right),
$$

where $i=1, \ldots, N$. Substituting (29) into (28), we get

$$
\lambda_{i}=A-\Lambda \varepsilon \widehat{\lambda_{i}}=A-\Lambda \varepsilon \cos \left(\frac{i \pi}{N}\right) .
$$

If we use (30) and assume that $A=(1-\varepsilon) \Lambda$, minimal and maximal values for the eigenvalues of the matrix $J$ can be found:

$$
\begin{aligned}
\lambda_{\min } & =A+\Lambda \varepsilon=(1-\varepsilon) \Lambda+\Lambda \varepsilon=\Lambda, \\
\lambda_{\max } & =A-\Lambda \varepsilon=(1-\varepsilon) \Lambda-\Lambda \varepsilon=(1-2 \varepsilon) \Lambda .
\end{aligned}
$$

The suffixes "min" and "max" in (31) and (32) depend on the values of the parameters $\varepsilon$ and $p$, and therefore they may be swapped. The system will be asymptotically stable for each lattice component when $\left|\lambda_{i}\right|<1$ for each $i$. In practice, it is sufficient to check the following conditions:

$$
\left\{\begin{array}{l}
\left|\lambda_{\min }\right|<1 \\
\left|\lambda_{\max }\right|<1
\end{array}\right.
$$

According to the Lyapunov method, since the linear approximation is asymptotically stable, the original nonlinear dynamic system will be asymptotically locally stable.

The nontrivial period-1 fixed point of the logistic map denoted by $x_{F}$ can be determined on the condition that $x_{t+1}=x_{t}=x_{F}$. Hence, using this and the logistic equation, it can be shown that $x_{F}=1-1 / p$. Then, the derivative of the logistic function $g(x)$ at the point $x_{F}$ can be written as

$$
\Lambda=g^{\prime}\left(x_{F}\right)=2-p .
$$

From (31)-34) we obtain

$$
\left\{\begin{aligned}
|2-p| & <1, \\
|(1-2 \varepsilon)(2-p)| & <1 .
\end{aligned}\right.
$$

According to 35, when the conditions

$$
\frac{p-3}{2 p-4}>\varepsilon>\frac{p-1}{2 p-4} \quad \text { for } \quad p \in(1,2)
$$

and

$$
\frac{p-3}{2 p-4}<\varepsilon<\frac{p-1}{2 p-4} \quad \text { for } \quad p \in(2,3)
$$

are met, the system is stable. Figure 4 illustrates the system behavior in time and space for $p=2.95$ and $\varepsilon=0.8$. Figure 5 shows the state trajectory for the component $n=25$ versus time. Based on the analysis of the system behavior shown in these two figures, it can be concluded that the values of all lattice states stabilize at the specified level.

The Lyapunov exponents are calculated by taking logarithms of the minimal and maximal of eigenvalues given 


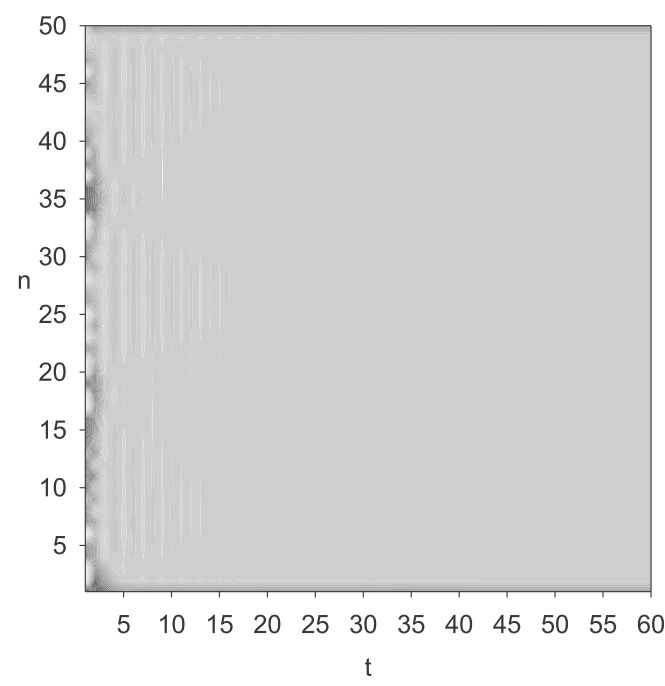

Fig. 4. Lattice behavior $x(n, t)$ for $p=2.95$ and $\varepsilon=0.8$.

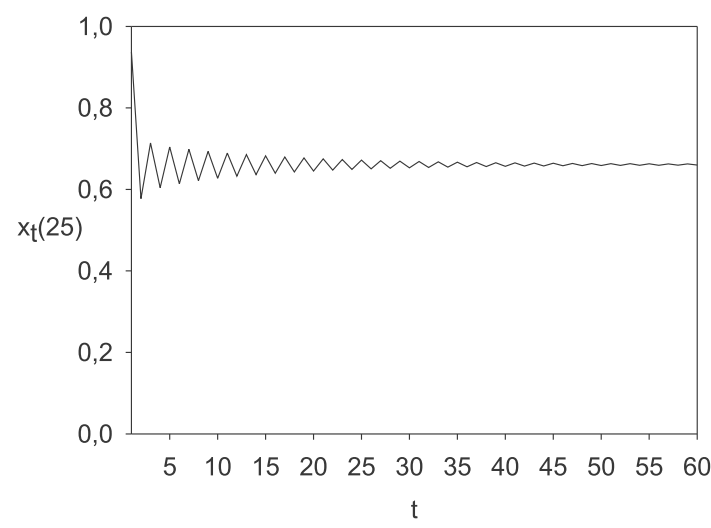

Fig. 5. State trajectory $x_{t}(25)$ for $p=2.95$ and $\varepsilon=0.8$.

by (31) and (32). In the case of CMLs, we have

$$
\begin{aligned}
& h_{\min }=\frac{1}{T} \sum_{t=1}^{T} \ln \mid g^{\prime}\left(x_{t}(n) \mid,\right. \\
& h_{\max }=\ln (|1-2 \varepsilon|)+\frac{1}{T} \sum_{t=1}^{T} \ln \mid g^{\prime}\left(x_{t}(n) \mid .\right.
\end{aligned}
$$

The element $\ln (|1-2 \varepsilon|)$ is related to the coupling with surrounding components in the CML and extends Eqn. (12), which defines the Lyapunov exponent for onedimensional, discrete equations.

Figures 6 and 7 show the distribution of the Lyapunov exponents and the net direction in space. As we can observe in Fig. 6, the Lyapunov exponents are negative for almost all lattice components, which indicates ordered system trajectories. Similar conclusions can be drawn from the distribution of the net direction phase (Fig. 7).

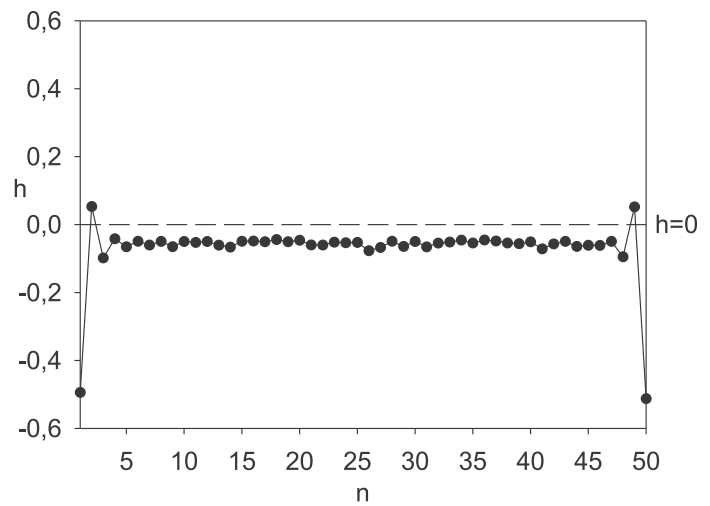

Fig. 6. Lyapunov exponents for all components $h(n)$ for $p=2.95$ and $\varepsilon=0.8$.

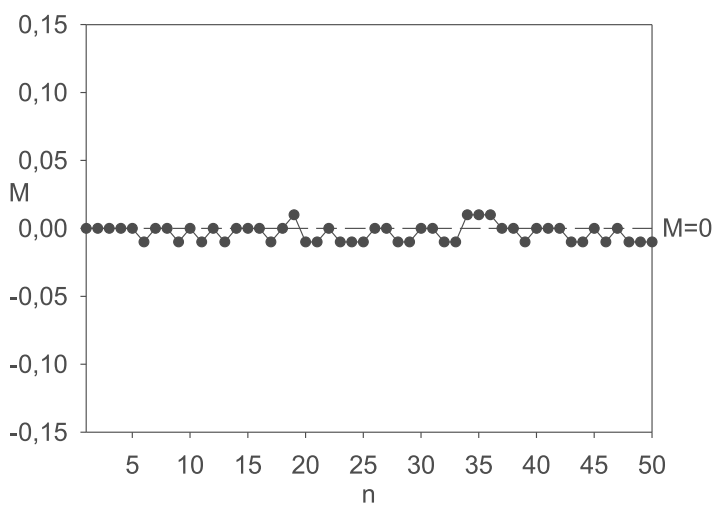

Fig. 7. Net direction phase for all components $M(n)$ for $p=$ 2.95 and $\varepsilon=0.8$.

The values of this performance index are similar for all lattice components and close to zero. This confirms the regular nature of system state trajectories and a symmetrical switching from increasing to decreasing state trajectories.

When the system parameters are set to $p=4$ and $\varepsilon=0.8$, the eigenvalues are

$$
\left\{\begin{aligned}
\lambda_{\min } & =(2-p)=-2, \\
\lambda_{\max } & =(1-2 \varepsilon)(2-p)=1.2 .
\end{aligned}\right.
$$

Both the values mentioned above are out of the range determined by (33), which means that the system is unstable. An irregular behavior of the system in time and space can be observed in the diagram shown in Fig. 8, The sensitivity to initial conditions for states in all lattice components is indicated by the distribution of the Lyapunov exponents (Fig. 9). The lack of symmetry in switching between increasing and decreasing trajectories is confirmed by the distribution of the net direction phase (Fig.10). Lyapunov exponents are positive for all lattice components, while 


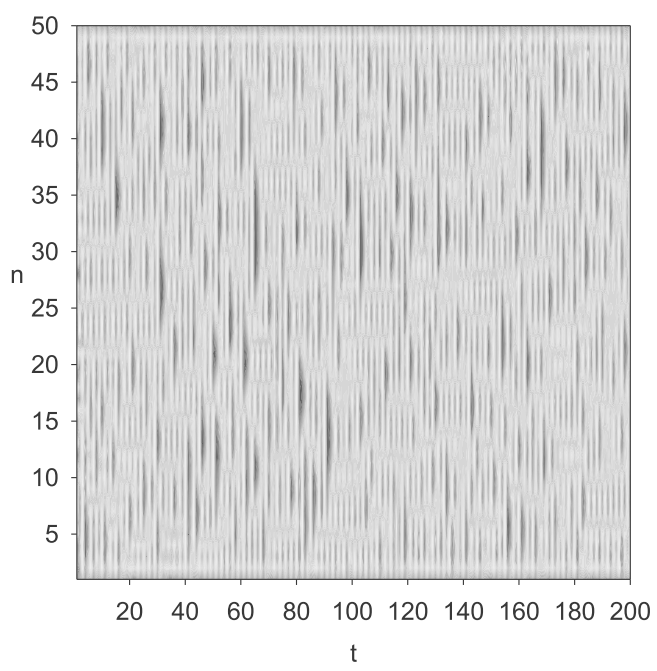

Fig. 8. Lattice behavior $x(n, t)$ for $p=4$ and $\varepsilon=0.8$.

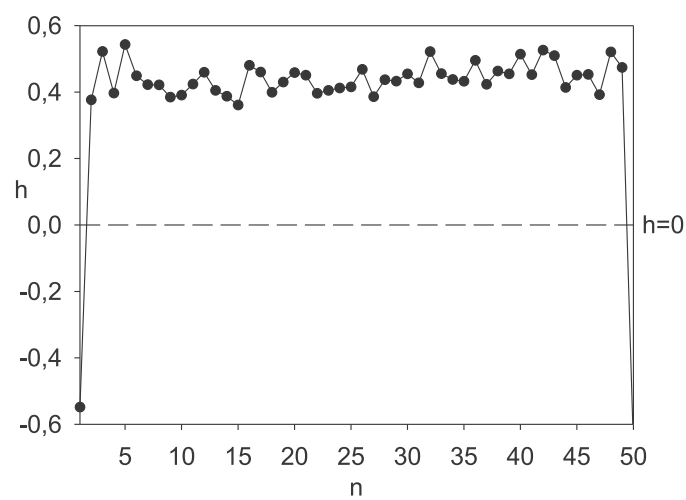

Fig. 9. Lyapunov exponents for all components $h(n)$ for $p=4$ and $\varepsilon=0.8$

the net direction phase is nonzero. Both the facts confirm the chaotic nature of the system behavior (verified by simulation).

\section{CML: Chaotic behavior}

Figures 2 and 8 contain diagrams which illustrate the CML behavior in time and space for parameters $\varepsilon=0.8$ and $p=4$. Based on these diagrams, it can only be stated that the system behavior is complex and disordered. Further conclusions can be drawn after the analysis of the Lyapunov exponents (Fig. 9) and the net direction phase (Fig. 10) for all lattice components. These diagrams confirm strong sensitivity to initial conditions and the lack of symmetry in state trajectories. The above mentioned conclusions, along with the fact that the logistic map (10) is used as the coupling function, indicate that state trajectories generated by all system components are chaotic.

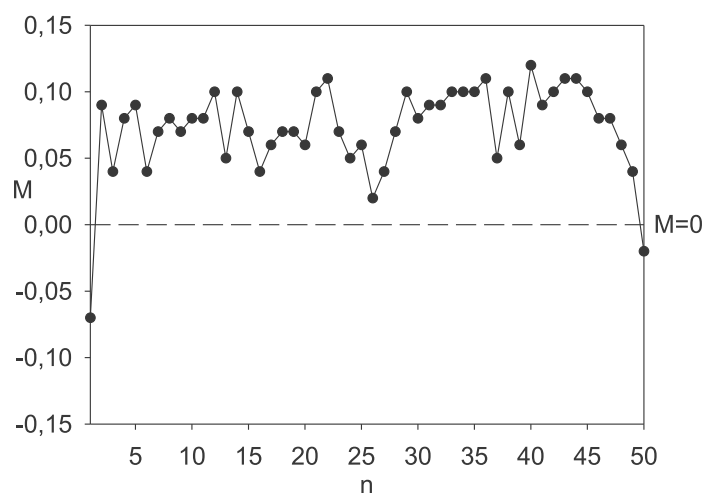

Fig. 10. Net direction phase for all components $M(n)$ for $p=4$ and $\varepsilon=0.8$.

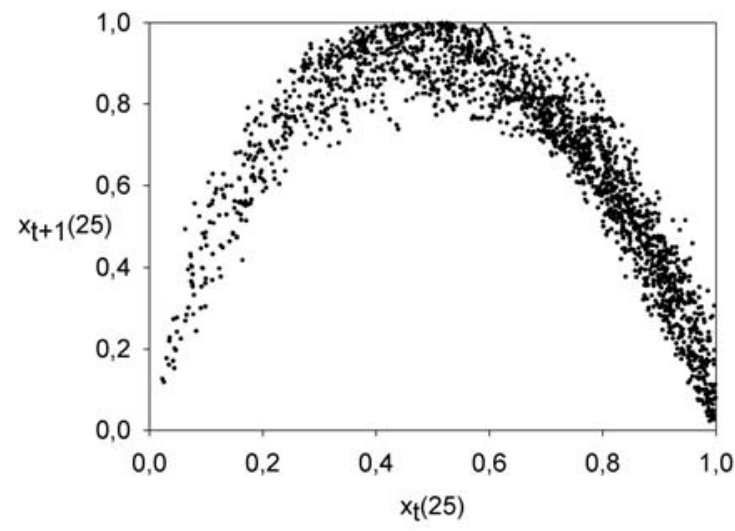

Fig. 11. Mapping which transforms the values of the components in time for the element $n=25$ for $\varepsilon=0.8$ and $p=4$.

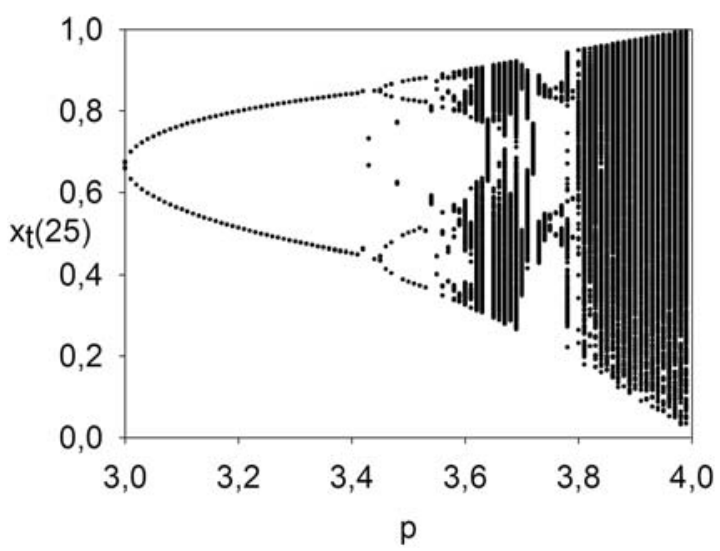

Fig. 12. Change in cell values in time versus the logistic equation parameter $p$ for $n=25$.

The main source of chaos in the CML is the chaotic nature of the logistic equation (10), which is used as the co- 


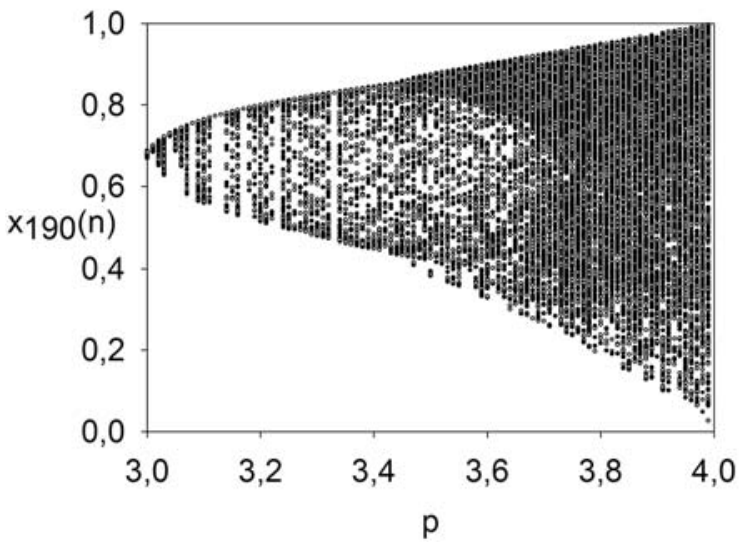

Fig. 13. Change in cell values in space versus the logistic equation parameter $p$ for $t=190$.

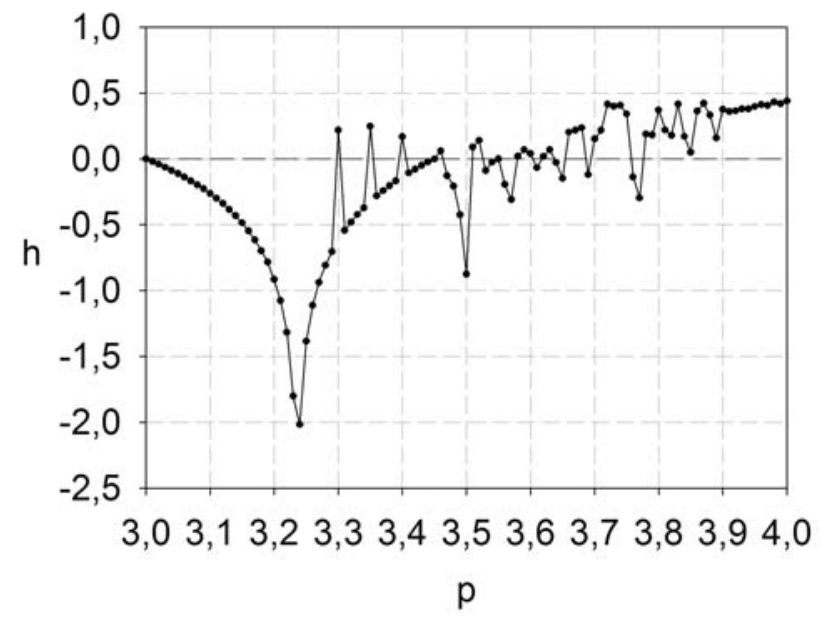

Fig. 14. Lyapunov exponent versus the parameter of the logistic equation $h(p)$ for a particular cell $(n=25)$.

upling function in the CML evolution equation (9). The chaotic behavior derived from the same lattice component but a previous time step is additionally strengthened by coupling with surrounding components. It also makes the mapping, which transforms the state values of time components, close to the logistic map, but the generated points are additionally scattered (Fig. 11). Moreover, such properties of the coupling function result in a chaotic behavior observed not only in time, but also in space. This is confirmed by diagrams which illustrate scenarios of transition to chaos in time and space shown in Figs. 12 and 13 The first of these figures shows the bifurcation of period doubling in time for the component $n=25$ when the parameter $p$ of the logistic map changes. Figure 13 shows a change in cell values in space for a fixed time moment $t=190$ versus the logistic equation parameter $p$. The scenario of transition to chaos in the space domain is more

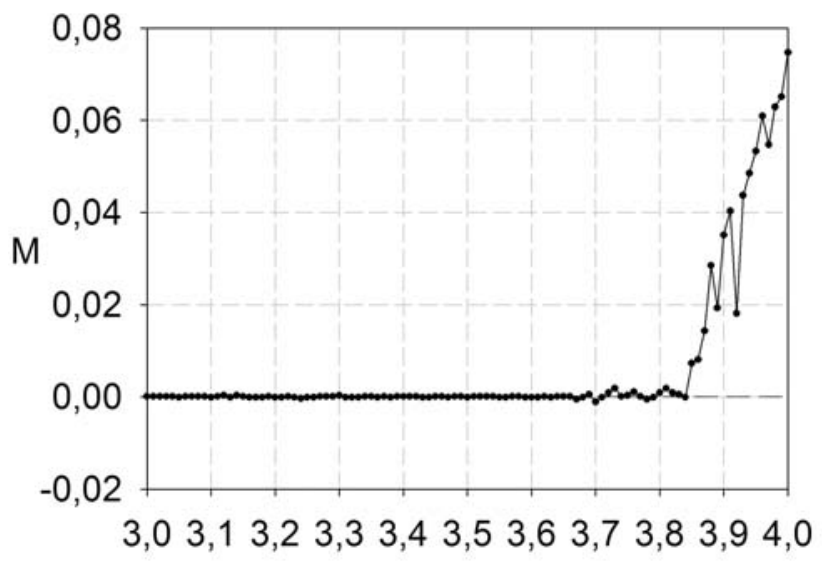

\section{$\mathrm{p}$}

Fig. 15. Net direction phase versus the parameter of the logistic equation $M(p)$ for a particular cell $(n=25)$.

complex and it is not about period doubling.

Based on these two diagrams, which present scenarios of transition to chaos in time and space domains, it can be concluded that, if we increase the value of the logistic equation parameter $p$, the set of possible state values becomes larger. This makes the system behavior more disordered and unpredictable. Figures 14 and 15 present the Lyapunov exponent and the net direction phase versus the logistic equation parameter $p$ for the lattice component $n=25$. As can be seen from Fig. 14, beyond some critical value of the parameter $p$, the values of the Lyapunov exponent remain mainly positive. This indicates the chaotic nature of system dynamics for this range. Trajectories, which are close to each other at the beginning, exhibit then an exponentially increased distance between them.

The diagram which shows the net direction phase versus the parameter $p$ of the logistic equation (Fig. 15b) additionally confirms that, beyond some value of parameter $p$, the system behaves irregularly. This corresponds to the range of the parameter $p$ where the values of the net direction phase are different from zero $\left(M_{n} \neq 0\right)$. This is connected with a nonsymmetric switching between the upward and downward direction phases of the trajectories. Then, the chaotic behavior of the system can be observed. Similar phenomena can be observed during the examination of Lyapunov exponents and net direction phases for all lattice components (Figs. 16 and 17). Both the diagrams show transition from a regular behavior $\left(h_{n}<0, M_{n} \approx 0\right)$ to a chaotic one $\left(h_{n}>0, M_{n} \neq 0\right)$.

Based on these two diagrams, it can be also stated that the system passes to a strongly chaotic behavior at about $p>3.6$. However, it should be added that also above this value of the parameter $p$, there are such ranges of time $t$ for some components, where the Lyapunov expo- 
nents are negative. This indicates the regular nature of the trajectories.

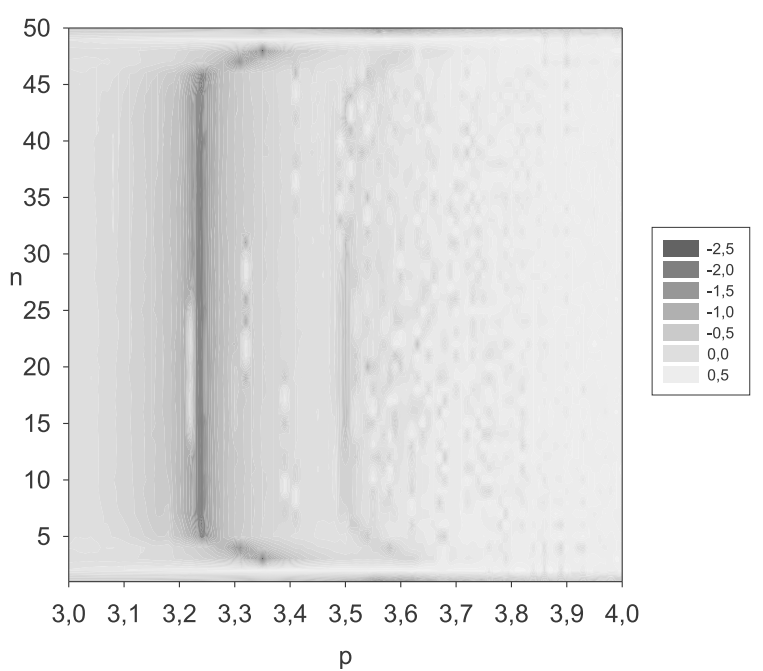

Fig. 16. Lyapunov exponents for all components $h_{n}$ versus the parameter $p$ of the logistic equation.

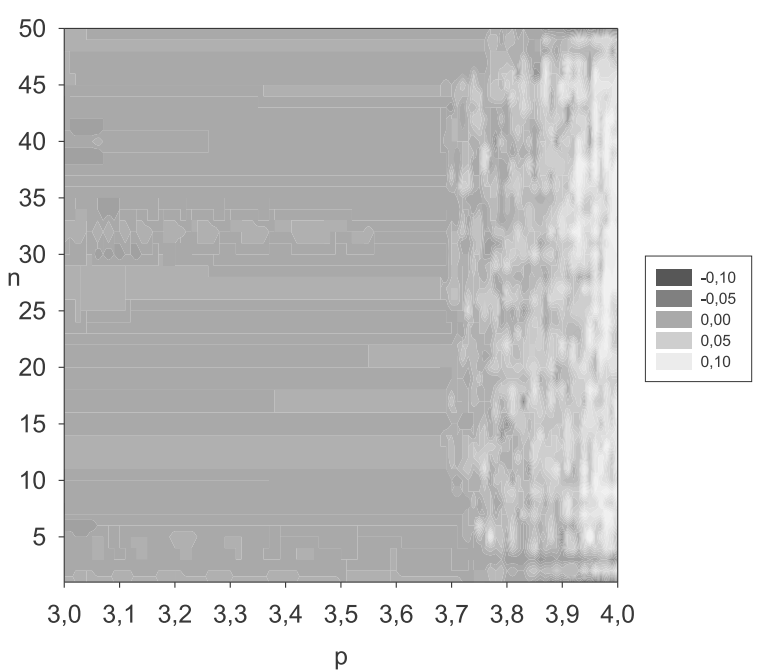

Fig. 17. Net direction phase for all components $M_{n}$ versus the parameter $p$ of the logistic equation.

To sum up the results given in this section, the CML is a mathematical model in which deterministic chaos can be observed in time and space. Chaos which is connected with the usage of the logistic map in the CML is additionally strengthened by coupling with surrounding components. Such a type of model can be used for modeling real systems with nonlinear interconnected elements. For instance, a CML was used to model the chain of vibrating objects in seismology (Greilich and Markus, 2003).

\section{CML: Example application}

Coupled map lattices, as has been illustrated in previous sections, are simple models with chaotic, spatiotemporal dynamics. They can be used for the modeling of real physical objects. As an example, a tubular reactor is presented in this section. The main aim of this object (Fig. 18) is to convert substance $A$ into product $B$. Due to the distributed nature of this object, the time variable $t \in[0, T]$ and the space variable $n \in[0, N]$ can be considered. Process variables are defined as functions of time and space:

- $x_{t}(n)$ : distributed state of the process, i.e., the concentration of raw material substance $A$ at time $t$ at point $n$ of the tubular reactor;

- $p_{t}(n)$ : distributed control of the process, i.e., the intensity of the heat source at time $t$ at point $n$ of the reactor;

- $u_{t}$ : concentrated boundary control of the process, i.e., the concentration of raw material substance $A$ at time $t$ in the input stream of the reactor;

- $v_{t}$ : concentrated flow control of the process, i.e., the flow intensity of the mixture through the reactor at time $t$.

There are two types of control in this system: concentrated and distributed. The physical system shown in Fig. 18 has a structure similar to the mathematical model presented in the previous sections. The lattice state at a specific time $t$, given by the vector (7), corresponds to the distributed state of the process in the tubular reactor. The vector of control parameters in the CML can be equated with a distributed control of the process in the tubular reactor. The concentrated control defined for the tubular reactor can also be performed in the CML by changing parameters for border components only. Hence, the structures of both the systems, the tubular reactor and the CML, are similar. The main issue is to select a proper coupling and local functions in the CML, which will assure that the model will fit to the modeled system.

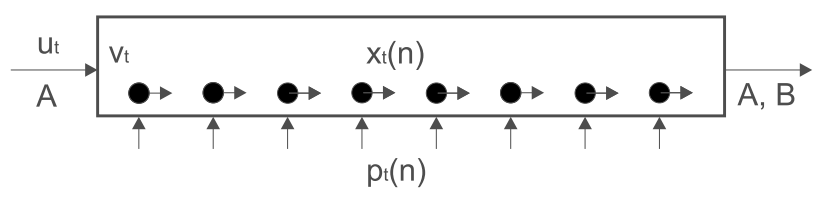

Fig. 18. Tubular reactor.

\section{CML in micro and macro scales}

Spatially distributed dynamic systems, such as the CML, can be observed in two scales: micro and macro. Similar to other chaotic systems, this one is also sensitive to changes in the initial conditions in micro scale. This means that a small change in the initial state in the particular component makes the system trajectory at this point of the lattice exponentially separate in time from the previous one. On 
the other hand, while observing collective behaviors of all lattice components in macro scale, it can be shown that a radical change in the initial conditions yields no change in the type of system behavior. This means that the state trajectory for a given component is dependent on initial conditions, but taking into consideration the whole system of collective components, it behaves very repetitively.

\section{Conclusions}

As was shown in this paper, the coupled map lattice is a simple mathematical model which generates complex, chaotic spatiotemporal behaviors for some range of its parameters. Based on the indirect Lyapunov method, the analysis of system stability was performed. Analytical results were compared with those obtained from numerical simulation. To review the existence of chaos during numerical studies, Lyapunov exponents and the net direction phase were introduced. Scenarios of transition to chaos in time and space domains were also provided. In addition, a real system which can be modeled by CMLs was presented. To sum up, the article described basic elements of the environment, which can be used for modeling spatiotemporal chaos. The presented model and tools can be also helpful while creating and examining algorithms of controlling chaos.

\section{References}

Alsing, P.M., Gavrielides, A. and Kovanis, V. (1994). Using neural networks for controling chaos, Physical Review E 49(2): 1225-1231.

Andrievskii, B.R. and Fradkov, A.L. (2003). Control of chaos: Methods and applications, Automation and Remote Control 64(5): 673-713.

Andrzejak, R.G., Lehnertz, K., Mormann, F., Rieke, C., David, P. and Elger, C.E. (2001). Indications of nonlinear deterministic and finite-dimensional structures in time series of brain electrical activity: Dependence on recording region and brain state, Physical Review E 64(1): 1-8.

Argoul, F., Arneodo, A., Richetti, P. and Roux, J.C. (1987). From quasiperiodicity to chaos in the Belousov-Zhabotinskii reaction, I: Experiment, Journal of Chemical Physics 86(6): 3325-3339.

Astakhov, V.V., Anishchenko, V.S. and Shabunin, A.V. (1995). Controlling spatiotemporal chaos in a chain of the coupled logostic maps, IEEE Transactions on Circuits and Systems 42(6): 352-357.

Auerbach, D. (1994). Controlling extended systems of chaotic elements, Physical Review Letters 72(8): 1184-1187.

Banerjee, S., Misra, A.P., Shukla, P.K. and Rondoni, L. (2010). Spatiotemporal chaos and the dynamics of coupled langmuir and ion-acoustic waves in plasmas, Physical Review E 81(1): 1-10.

Beck, O., Amann, A., Scholl, E., Socolar, J.E.S. and Just, W. (2002). Comparison of time-delay feedback schemes for spatiotemporal control of chaos in a reaction-diffusion system with global coupling, Physical Review E 66(1): 1-6.

Boukabou, A. and Mansouri, N. (2005). Predictive control of higher dimensional chaos, Nonlinear Phenomena in Complex Systems 8(3): 258-265.

Chen, G. and Dong, X. (1993). On feedback control of chaotic continuous-time systems, IEEE Transactions on Circuits and Systems 40(9): 591-601.

Chopard, B., Dupuis, A., Masselot, A. and Luthi, P. (2002). Cellular automata and lattice Boltzmann techniques: An approach to model and simulate complex systems, Advances in Complex Systems 5(2): 103-246.

Chui, S.T. and Ma, K.B. (1982). Nature of some chaotic states for Duffing's equation, Physical Review A 26(4) 2262-2265.

Córdoba, A., Lemos, M. C. and Jiménez-Morales, F. (2006). Periodical forcing for the control of chaos in a chemical reaction, Journal of Chemical Physics 124(1): 1-6.

Crutchfield, J.P. and Kaneko, K. (1987). Directions in Chaos. Phenomenology of Spatio-Temporal Chaos, World Scientific Publishing Co., Singapore.

Dressler, U. and Nitsche, G. (1992). Controlling chaos using time delay coordinates, Physical Review Letters 68(1): $1-4$

Gautama, T., Mandic, D.P. and Hulle, M.M.V. (2003). Indications of nonlinear structures in brain electrical activity, Physical Review E 67(1): 1-5.

Govindan, R.B., Narayanan, K. and Gopinathan, M.S. (1998) On the evidence of deterministic chaos in ECG: Surrogate and predictability analysis, Chaos 8(2): 495-502.

Greilich, A. and Markus, M. (2003). Correlation of entropy with optimal pinning density for the control of spatiotemporal chaos, Nonlinear Phenomena in Complex Systems 6(1): 541-546.

Gunaratne, G.H., Lisnay, P.S. and Vinson, M.J. (1989). Chaos beyond onset: A comparison of theory and experiment, Physical Review Letters 63(1): 1-4.

Held, G.A., Jeffries, C. and Haller, E.E. (1984). Observation of chaotic behavior in an electron-hole plasma in GE, Physical Review Letters 52(12): 1037-1040.

Jacewicz, P. (2002). Model Analysis and Synthesis of Complex Physical Systems Using Cellular Automata, Ph.D. thesis, University of Zielona Góra, Zielona Góra.

Kaneko, K. (1990). Simulating Physics with Coupled Map Lattices, World Scientific Publishing Co., Singapore.

Korus, L. (2007). Alternative methods of wave motion modelling, in B. Apolloni, R.J. Howlett and L. Jain (Eds.) Knowledge-Based Intelligent Information and Engineering Systems: KES2007/WIRN2007, Part 1, Lecture Notes in Artificial Intelligence, Vol. 4692, Springer-Verlag, Berlin/Heidelberg, pp. 335-345.

Kwon, Y.S., Ham, S.W. and Lee, K.K. (1997). Analysis of minimal pinning density for controlling spatiotemporal chaos of a coupled map lattice, Physical Review E 55(2) 2009-2012. 
Langenberg, J., Pfister, G. and Abshagen, J. (2004). Chaos from Hopf bifurcation in a fluid flow experiment, Physical Review E 70(4): 046209.

Mihaliuk, E., Sakurai, T., Chirila, F. and Showalter, K. (2002). Feedback stabilization of unstable propagating waves, Physical Review E 65(6): 065602.

Ott, E. (2002). Chaos in Dynamical Systems, Cambridge University Press, Cambridge.

Ott, E., Grebogi, C. and Yorke, J.A. (1990). Controlling chaos, Physical Review Letters 64(11): 1196-1199.

Parekh, N., Parthasarathy, S. and Sinha, S. (1998). Global and local control of spatiotemporal chaos in coupled map lattice, Physical Review Letters 81(7): 1401-1404.

Parmananda, P. (1997). Controlling turbulence in coupled map lattice systems using feedback techniques, Physical Review E 56(1): 239-244.

Procaccia, I. and Meron, E. (1986). Low-dimensional chaos in surface waves: Theoretical analysis of an experiment, Physical Review A 34(4): 3221-3237.

Pyragas, K. (2001). Control of chaos via an unstable delayed feedback controller, Physical Review Letters 86(11): 2265-2268.

Singer, J., Wang, Y. and Haim, H. B. (1991). Controlling a chaotic system, Physical Review Letters 66(9): 1123-1125.

Used, J. and Martin, J.C. (2010). Multiple topological structures of chaotic attractors ruling the emission of a driven laser, Physical Review E 82(1): 016218.
Wei, W., Zonghua, L. and Bambi, H. (2000). Phase order in chaotic maps and in coupled map lattices, Physical Review Letters 84(12): 2610-2613.

Weimar, J.R. (1997). Simulation with Cellular Automata, Logos Verlang Berlin, Berlin.

Yamada, T. and Graham, R. (1980). Chaos in a laser system under a modulated external field, Physical Review Letters 45(16): 1322-1324.

Yim, G., Ryu, J., Park, Y., Rim, S., Lee, S., Kye, W. and Kim, C. (2004). Chaotic behaviors of operational amplifiers, Physical Review E 69(4): 045201.

Zhu, K. and Chen, T. (2001). Controlling spatiotemporal chaos in coupled map lattice, Physical Review E 63(3): 067201.

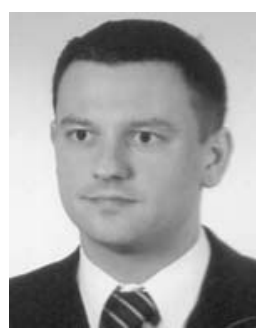

Łukasz Korus was born in 1978 in Poland. He received M.Sc. degrees in automatic control and robotics as well as computer engineering from the Wrocław University of Technology, Poland, in 2003 and 2006, respectively. In 2009, he received the Ph.D. degree in automatic control and robotics from the same university. Since 2009 he has been an assistant professor at the Institute of Informatics, Automatics and Robotics of the Wrocław University of Technology. His research interests include chaos and control theories, and their application to the control of chaotic behavior in spatially distributed systems.

Received: 11 April 2010

Revised: 6 August 2010

Re-revised: 17 September 2010 